\title{
Inhibitory role of TRIP-Br1 oncoprotein in hypoxia-induced apoptosis in breast cancer cell lines
}

\author{
CHENGPING LI ${ }^{1,2}$, SAMIL JUNG ${ }^{1}$, YOUNG YANG ${ }^{1}$, KEUN-IL KIM $^{1}$, JONG-SEOK LIM ${ }^{1}$, \\ CHUNG-IL CHEON ${ }^{1}$ and MYEONG-SOK LEE ${ }^{1}$ \\ ${ }^{1}$ Department of Biological Science, Sookmyung Women's University, Seoul 140-742, Republic of Korea \\ Received January 19, 2016; Accepted February 18, 2016
}

DOI: 10.3892/ijo.2016.3454

\begin{abstract}
TRIP-Br1 oncoprotein is known to be involved in many vital cellular functions. In this study, we examined the role of TRIP-Br1 in hypoxia-induced cell death. Exposure to the overcrowded and $\mathrm{CoCl}_{2}$-induced hypoxic conditions increased TRIP-Br1 expression at the protein level in six breast cancer cell lines (MCF7, MDA-MB-231, T47D, Hs578D, BT549, and MDA-MB-435) but resulted in no significant change in three normal cell lines (MCF10A, MEF and NIH3T3). Our result revealed that $\mathrm{CoCl}_{2}$-induced hypoxia stimulated apoptosis and autophagy, in which TRIP$\mathrm{Br} 1$ expression was found to be upregulated. Interestingly, TRIP-Br1 silencing in the MCF7 and MDA-MB-231 cancer cells accelerated apoptosis and destabilization of XIAP under the $\mathrm{CoCl}_{2}$-induced hypoxic condition, implying that TRIP-Br1 may render cancer cells resistant to apoptosis through the stabilization of XIAP. We also propose that TRIP-Br1 seems to be upregulated at least partly as a result of the inhibition of PI3K/AKT signaling pathway and the overexpression of HIF-1 $\alpha$. In conclusion, our findings suggest that TRIP-Brl functions as an oncogenic protein by providing cancer cells resistance to the hypoxia-induced cell death.
\end{abstract}

Correspondence to: Professor Myeong-Sok Lee, Department of Biological Science, Sookmyung Women's University, Seoul 140-742, Republic of Korea

E-mail: mslee@sookmyung.ac.kr

Present address: ${ }^{2}$ College of Life Science, Liaocheng University, Liaocheng, Shandong 252000, P.R. China

\begin{abstract}
Abbreviations: TRIP-Br1, transcriptional regulator interacting with the PHD-bromodomain 1; TRIP-Br3, transcriptional regulator interacting with the PHD-bromodomain 3; XIAP, X-linked inhibitor of apoptosis protein; PI3K, phosphoinositol-3-kinase; HIF-1 $\alpha$, hypoxia-inducible factor $1 \alpha$
\end{abstract}

Key words: transcriptional regulator interacting with the PHDbromodomain 1, hypoxia, cell death, apoptosis, X-linked inhibitor of apoptosis protein

\section{Introduction}

The environment surrounding cells or tissues is considered to be an important factor affecting their biological progress, including cell growth, proliferation, tumorigenesis, and cell death. During tumorigenesis, cancer cells are under extremely stressful conditions (e.g., nutrient starvation, acidic, or hypoxic conditions) because of their uncontrolled growth and proliferation (1-3). Hypoxia, a condition of a poor oxygen supply, plays a vital role in tumorigenesis, angiogenesis, metabolism, proliferation, metastasis, and cell death (4-9). When cells are initially exposed to hypoxia, they stimulate particular adaptive responses such as autophagy, which regulate a series of physiological and cellular mechanisms necessary for survival (10-15). Further severe hypoxia eventually results in apoptotic cell death (14,16-18). Although cancer cells are usually much more sensitive than normal cells to such stress, they seem to have developed adaptive mechanisms at different molecular levels to withstand this extreme environmental condition and to delay or suppress cell death, thus aggravating tumorigenesis (19-21). Therefore, the precise understanding of the molecular mechanism of hypoxia-induced cancer cell growth and death appears to be very important in cancer research and treatment.

In an effort to identify the proteins responsible for the resistance of cancer cells to hypoxia-induced cell death, we initially focused on TRIP-Br1, the transcriptional regulator interacting with PHD-bromodomain 1 (also known as SERTAD1, p34SEI-1 or SEI-1), a member of the TRIP-Br family. TRIP-Br1 is known to be involved in various important biological functions, such as transcription, cell cycle progression, metabolism, metastasis, tumorigenesis, and cell death (22-31). However, little is known about its cellular function under hypoxic condition. It is now widely accepted that many oncoproteins suppress autophagy and apoptosis, whereas tumor suppressors mostly induce them $(32,33)$. In our previous study, we showed that the expression of TRIP-Br1 oncoprotein significantly increased in response to nutrient starvation, rending cancer cells resistant to cell death by suppressing three types of representative programed cell deaths (type I apoptosis, type II autophagy-induced cell death, and type III/IV necrosis/necroptosis) (34). Therefore, it was hypothesize that TRIP-Br1 might inhibit cell death under hypoxic condition. 
In this study, we attempted to clarify how TRIP-Br1 contributes to the survival of cancer cells under hypoxic condition during tumor growth.

\section{Materials and methods}

Cell lines, cell cultures, and materials. Six breast cancer cell lines (MCF7, MDA-MB-231, T47D, Hs578D, BT549, and MDA-MB-435) and two fibroblast normal cell lines (MEF and NIH3T3) were cultured in Dulbecco's modified Eagle's medium (DMEM; Welgene Inc., Daegu, Korea) supplemented with 10\% fetal bovine serum (FBS; Gibco BRL, Carlsbad, CA, USA) and $1 \%$ antibiotic-antimycotic (Gibco BRL). MCF10A normal breast epithelial cells were grown in DMEM/F12 medium (Invitrogen, Carlsbad, CA, USA, cat. 11330-032) supplemented with $20 \mathrm{ng} / \mathrm{ml}$ of epithelial growth factor (EGF; Sigma-Aldrich, cat. E9644), $100 \mathrm{ng} / \mathrm{ml}$ of cholera toxin (Sigma-Aldrich, cat. C-8052), $10 \mu \mathrm{g} / \mathrm{ml}$ of insulin (Sigma-Aldrich, cat. I-9278), $0.5 \mathrm{mg} / \mathrm{ml}$ of hydrocortisone (Sigma-Aldrich, cat. H-0888), 5\% horse serum (Invitrogen, cat. 16050-122), and 1\% antibioticantimycotic. All cells were cultured at $37^{\circ} \mathrm{C}$ in a humidified atmosphere composed of $5 \% \mathrm{CO}_{2}$. Cell lines were purchased from the American Type Culture Collection (ATCC). Cobalt chloride $\left(\mathrm{CoCl}_{2}\right)$ and LY294004 were purchased from SigmaAldrich (cat. STBC9672V) and Calbiochem, San Diego, CA, USA (cat. 440202), respectively.

Western blot analysis. Immunoblotting analysis was performed as previously described (35). Antibodies used in this study were TRIP-Br1 (Enzo Life Sciences, cat. ALX-804-645), TRIP-Br3 (Abcam, cat. ab107944), HIF-1 $\alpha$ (Cell Signaling Technology, cat. 3716S), PARP (Cell Signaling Technology, cat. 9542), Bax (Santa Cruz Biotechnology, cat. sc-20067), XIAP (Cell Signaling Technology, cat. 2042), SQSTM1/p62 (Cell Signaling Technology, cat. 5114S), LC3 (Enzo Life Sciences, cat. ALX-803-082), and $\gamma$-tubulin (Santa Cruz Biotechnology, cat. sc-7396).

Reverse transcription polymerase chain reaction (RT-PCR) analysis. Total RNA was extracted from MCF7, MDA-MB231, and MCF10A cells by using an RNeasy mini kit (Qiagen, Hilden, Germany). For reverse transcription, $1 \mu \mathrm{g}$ of RNA from each sample was subjected to cDNA synthesis using the TOPscript $^{\mathrm{TM}}$ RT2XPreMIXkit(Enzynomics, cat. RT203-50-F, Korea) according to the manufacturer's instructions. Each gene product was amplified using $10 \mathrm{ng}$ of cDNA, the corresponding pair of primers, and an AccuPower PCR PreMix system (Bioneer, cat. K-2016, Korea), in which the $\beta$-actin gene product was used as an internal control. The oligonucleotide sequences for RT-PCR analysis were pRT-HIF-1 $\alpha-\mathrm{F} / \mathrm{R}$ : 5'-CATGGAAGGTATTGCACTGC-3'/5'-TGGCAAGCATC CTGTACTGT-3'; pRT-TRIP-Br1-F/R: 5'-AGGACCTCAGC CACATTGAG-3'/5'-GGTGCCCAAAGTTCATTGTC-3'; pRT-TRIP-Br3-F/R: 5'-CTGGTGAAGTTGCAGCTTTG-3' 5'-GGCAAAGGTCAGAAACTGGA-3'; pRT- $\beta$-actin-F/R: 5'-AGGTCGGAGTCAACGGATTTG-3'/5'-GTGATGGCAT GGACTGTGGT3'.

Suppression of TRIP-Brl gene and overexpression of TRIPBrl and HIF-1 $\alpha$ genes. To repress TRIP-Brl expression, cells were transfected with scrambled small interfering RNA (scRNA) or TRIP-Brl silencing siRNA (siTRIP-Brl) (Santa Cruz Biotechnology, cat. sc-62988). These cells were then incubated in Opti-MEM (Invitrogen, cat. 31985) at $37^{\circ} \mathrm{C}$ for $6 \mathrm{~h}$ and the transfection medium was replaced with fresh growth medium. For ectopic overexpression of TRIP-Br1 and HIF-1 $\alpha$, MCF7 and MDA-MB-231 cells were transfected with $8 \mu \mathrm{g}$ of TRIP-Brl (pTRIP-Br1) or HIF-1 $\alpha$ (pHIF-1 $\alpha$ ) overexpressing plasmids and their corresponding control vectors (pEGFP or pCMV-tag2B) by using Lipofectamine 2000 (Invitrogen, cat. 52887, Korea) for $48 \mathrm{~h}$. The pTRIP-Brl and pHIF-1 $\alpha$ plasmids were kindly provided by Dr Rikiro Fukunaga (Osaka University, Japan) and Dr Young Yang (Sookmyung Women's University, Republic of Korea), respectively.

Analysis of apoptosis and autophagy. Apoptosis and autophagy were mainly analyzed by employing western blotting with corresponding markers or regulatory proteins: PARP, Bax, and XIAP for apoptosis; SQSTM1/p62 and LC3 for autophagy. Cell viability was evaluated by means of the MTT assay following our previous method (36).

\section{Results}

Upregulated TRIP-Brl expression in overcrowded and hypoxic conditions. During tumorigenesis, the uncontrolled growth of cancer cells gives rise to oxygen deficiency and nutrient starvation and eventually cancer cells are under much more stressful condition compared to normal cells. To elucidate how cancer cells overcome these stressful environments and continue growing, we cultured six breast cancer cell lines (MCF7, MDA-MB-231, T47D, Hs578D, BT549, and MDA-MB-435) and normal cell lines (MCF10A, MEF, and NIH3T3) in complete media until cell densities reached $\sim 80 \%$ confluence to serve as the normal control (NC) or until the cells reached a very high cell density and became overcrowded (OC). Our previous data revealed that overcrowded condition increased TRIP-Br1, but decreased TRIP-Br3 at the protein level $(34,37)$. We proposed that a balance between TRIP-Brl and TRIP-Br3 levels seems to be crucial to cell survival and death $(34,37)$.

TRIP-Br3 (also known as SEI-3/CDCA4/Hepp) is a putative tumor suppressor whereas TRIP-Brl is an oncoprotein even though both of them belong to the same TRIP-Br family $(35,38-40)$. Therefore, the levels of TRIP-Br1 and TRIP-Br3 expression were determined under condition of overcrowding. Noteworthy, TRIP-Br1 expression increased significantly in all six cancer cell lines but only slightly in the three normal cell lines, which were not overcrowded, probably because their growth and proliferation were controlled (i.e., in a monolayer with $\sim 90 \%$ confluence) (Fig. 1A). In contrast, TRIP-Br3 expression decreased in both the cancer cells and the normal cells, in which TRIP-Br3 expression decreased to a greater extent in the normal cells than the cancer cells (Fig. 1A). In an effort to find what kind of stressful condition(s) is responsible for the TRIP-Br1 upregulation and TRIP-Br3 downregulation under the overcrowded condition, we previously showed that decreased nutrients (such as serum, glucose, and amino acids) affect TRIP-Br1 and TRIP-Br3 expression $(34,37)$. An overcrowded environment leads to another 
A

\begin{tabular}{|c|c|c|c|c|c|c|c|c|c|c|}
\hline \multicolumn{11}{|c|}{ Breast cancer cell lines } \\
\hline \multirow{2}{*}{$\frac{\text { MCF7 }}{\text { NC OC }}$} & \multicolumn{2}{|c|}{ MDA-MB-23 } & \multicolumn{2}{|c|}{ T47D } & \multicolumn{2}{|c|}{ Hs578D } & \multicolumn{2}{|c|}{ BT549 } & \multicolumn{2}{|c|}{ MDA-MB-43 } \\
\hline & NC & OC & NC & OC & $\mathrm{NC}$ & OC & NC & OC & $\mathrm{NC}$ & OC \\
\hline-1 & - & - & - & $=$ & - & 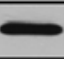 & - & - & - & - \\
\hline-- & - & - & 5 & - & & - & $=$ & - & & -1 \\
\hline$\underline{z}$ & - & $\Rightarrow$ & teis & $-\infty$ & & 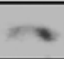 & & & & $=0$ \\
\hline-- & - & - & $=$ & - & & - & $\longrightarrow$ & - & - & - \\
\hline
\end{tabular}

B

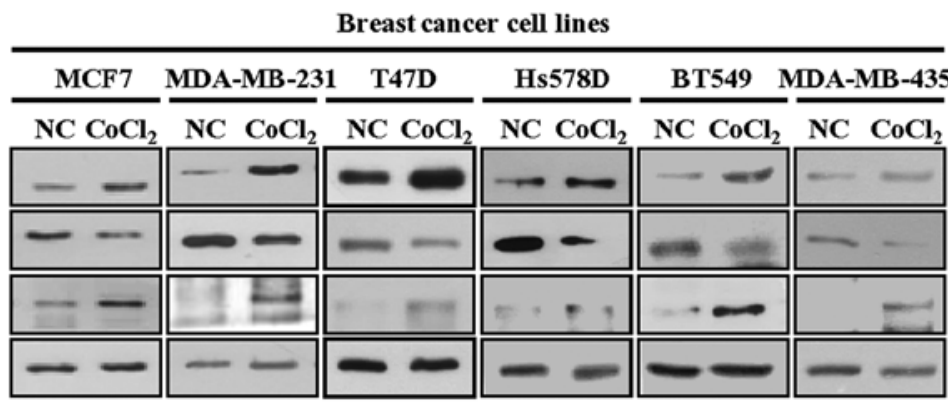

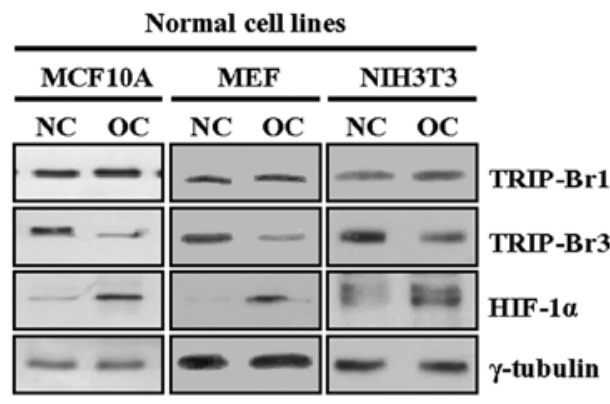

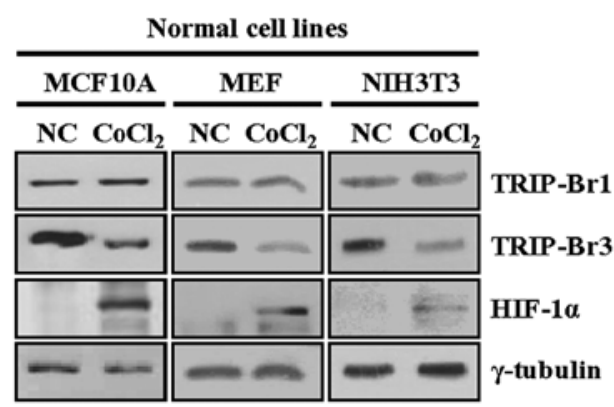

C
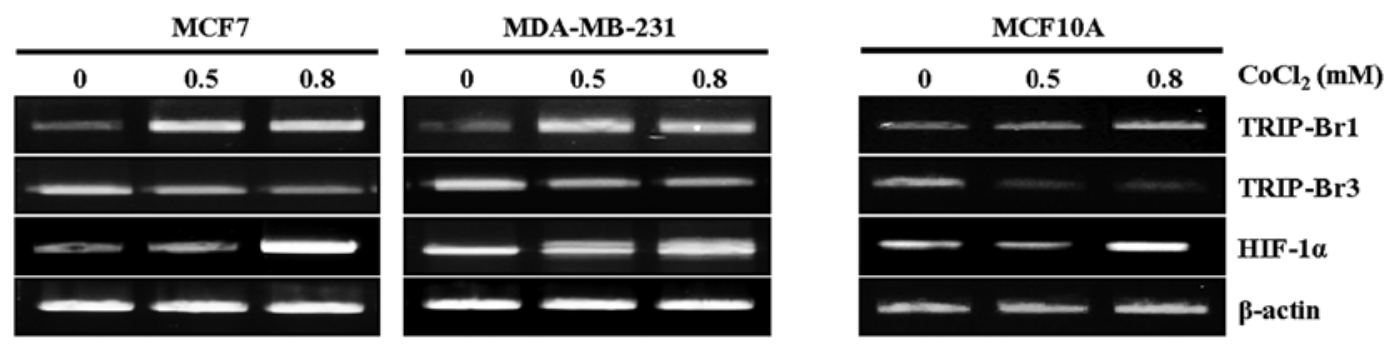

Figure 1. Upregulated TRIP-Br1 expression in overcrowded and hypoxic conditions. (A) Six breast cancer cell lines (MCF7, MDA-MB-231, T47D, Hs578D, BT549, and MDA-MB-435) and three normal cell lines (MCF10A, MEF, and NIH3T3) were cultured in complete media until the cells either reached 80\% confluence (indicated as ' $\mathrm{NC}$ ') or were overcrowded at high levels of cell confluence by being cultured in a complete medium for long time (indicated as 'OC'). TRIP-Br1 and TRIP-Br3 expression levels were checked by means of western blot analysis, in which HIF-1 $\alpha$ was used as a marker for hypoxic condition. (B) The six breast cancer cell lines and three normal cell lines were incubated for $24 \mathrm{~h}$ in growth media under either normoxic or hypoxic ( $0.8 \mathrm{mM}$ of $\mathrm{CoCl}_{2}$ ) conditions and levels of TRIP-Br1 and TRIP-Br3 expression were measured at the protein level. (C) MCF7 and MDA-MB-231 breast cancer cells and MCF10A normal cells were cultured in media with the concentration of $\mathrm{CoCl}_{2}$ as indicated for $24 \mathrm{~h}$, after which TRIP-Br1 and TRIP-Br3 expression levels were measured at the transcriptional level by using RT-PCR, with $\beta$-actin used as the internal control.

type of extreme stress than that caused by hypoxia, as well as nutrient starvation. Our data showed that HIF-1 $\alpha$ expression, a marker of hypoxia, increased as a result of overcrowding, suggesting the presence of hypoxia in an overcrowded environment (Fig. 1A).

Assuming that TRIP-Br1 and TRIP-Br3 expression might be affected at least in part by overcrowding-induced hypoxia, we examined their expression levels under cobalt chloride $\left(\mathrm{CoCl}_{2}\right)$-mediated hypoxic condition. The same cell lines were treated with $0.8 \mathrm{mM}$ of $\mathrm{CoCl}_{2}$, a well-established chemical inducer of hypoxia-like responses (4). TRIP-Br1 expression was significantly increased in all six cancer cell lines, but no significant change was detected in the three normal cell lines when they were exposed to $\mathrm{CoCl}_{2}$-generated hypoxia (Fig. 1B). In contrast, TRIP-Br3 expression decreased in both cancer cells and normal cells (Fig. 1B). These results suggest that the hypoxic condition is responsible for TRIP-Brl upregulation and TRIP-Br3 downregulation. In a further study, the expression levels of TRIP-Br1 and TRIP-Br3 in response to hypoxia were also tested at the transcriptional level and similar result was obtained (Fig. 1C).
Taken together, our data strongly suggest that hypoxic condition induces TRIP-Br1 upregulation and TRIP-Br3 downregulation at both the transcriptional and protein levels.

Inhibitory role of TRIP-Brl in hypoxia-induced cell death. TRIP-Br1 expression significantly increased in the cancer cell lines but not in the normal cell lines under $\mathrm{CoCl}_{2}$-generated hypoxic condition. We then sought to determine the cellular function of TRIP-Brl upregulation. Previously, we showed that TRIP-Br1 functions as an oncoprotein by inhibiting cell death in response to anticancer drug treatment and nutrient/serum starvation $(25,34)$. Considering these results, it was presumed that TRIP-Br1 might function in the same way under hypoxic condition. This hypothesis was tested by determining the effect of TRIP-Brl on cell death.

Among the above cell lines, we chose three major representative cell lines, MCF7, MDA-MB-231, and normal MCF10A for further studies. Since all of the cell lines we used showed very similar results in the expression and functions of TRIPBrl upon treatment of various stresses including anticancer drug, nutrient depletion, and hypoxia $(31,34)$, we chose three 
A

0

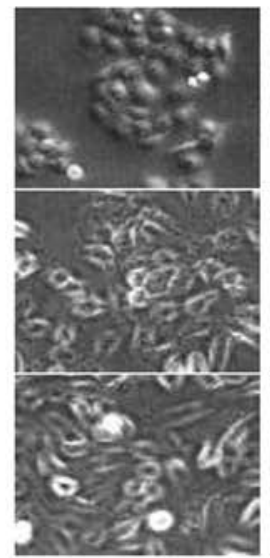

0.5

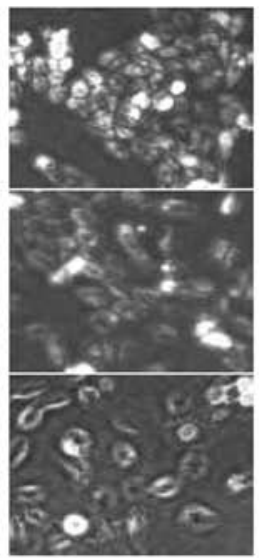

0.8

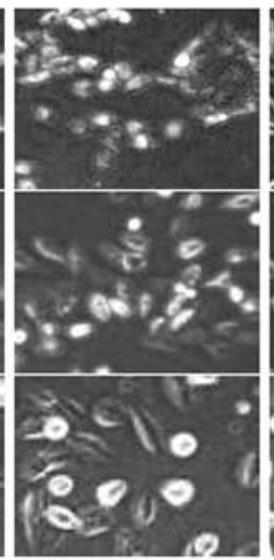

1.0

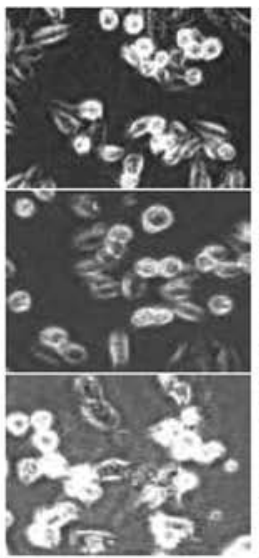

1.5

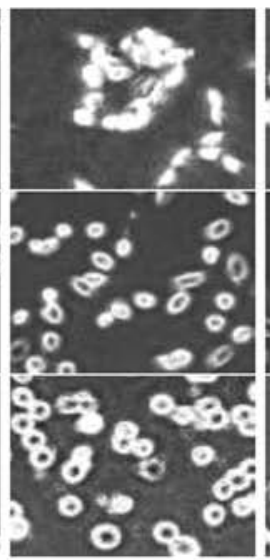

2.0

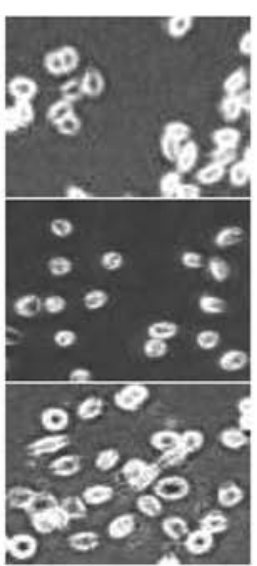

$\mathrm{CoCl}_{2}(\mathrm{mM})$

MCF7

MDA-MB-231

MCF10A

\section{MCF10A}

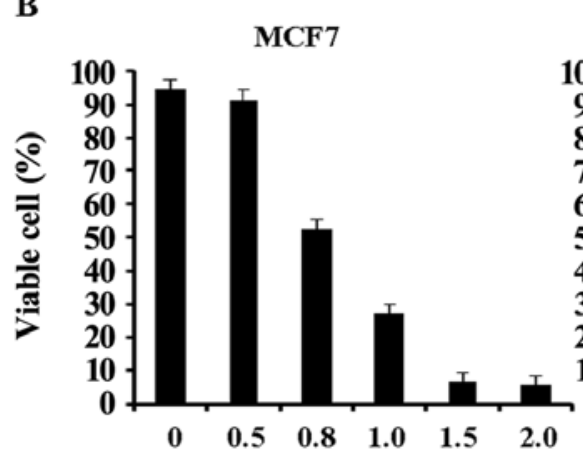

MDA-MB-231
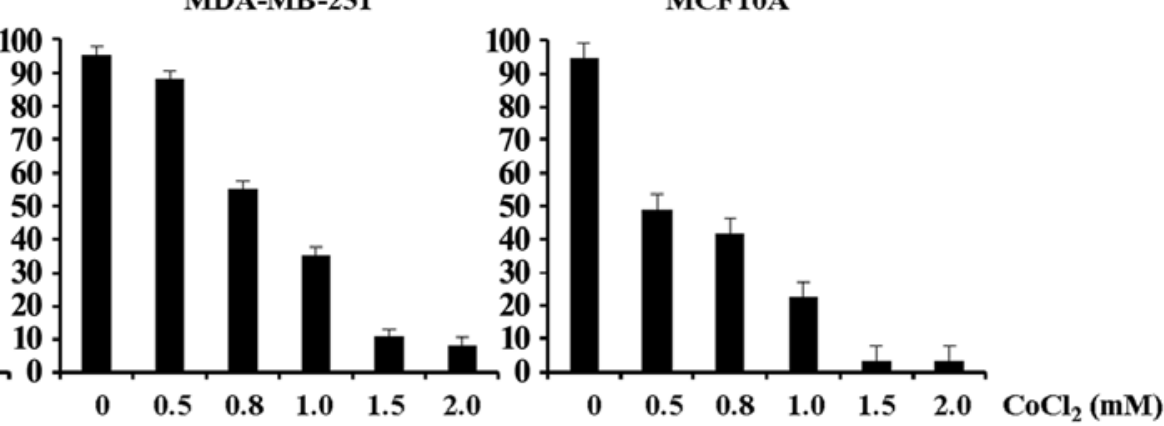

C

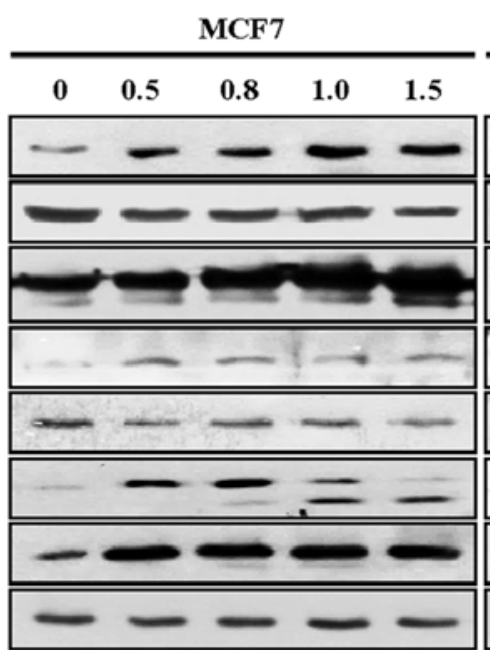

MDA-MB-231

MCF10A
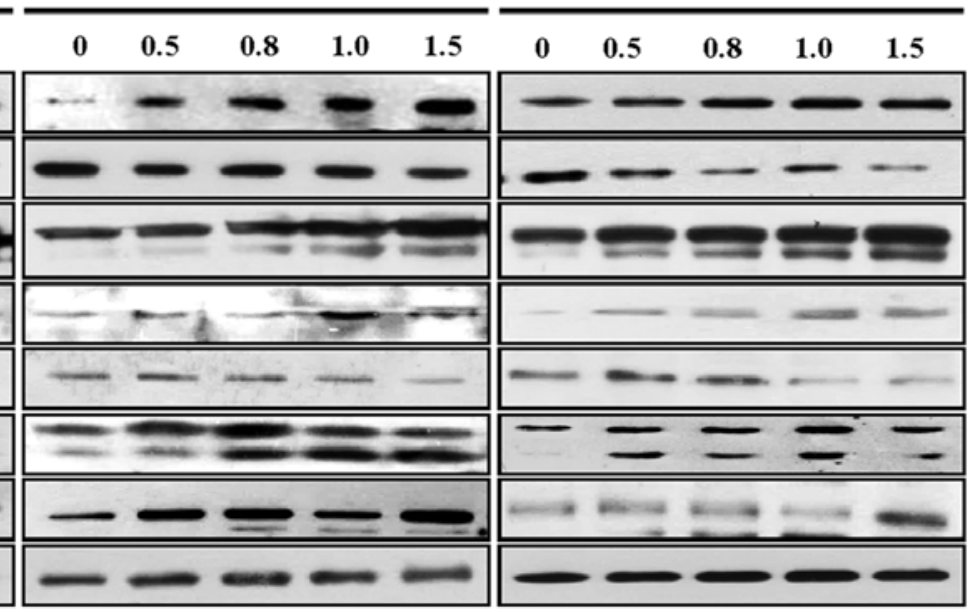

$\mathrm{CoCl}_{2}(\mathrm{mM})$

TRIP-Br1

TRIP-Br3

PARP

Cleaved PARP

Bax

SQSTM1/p62

LC3-I

LC3-II

HIF-1 $\alpha$

$\gamma$-tubulin

Figure 2. Inhibitory role of TRIP-Br1 on hypoxia-induced apoptosis. MCF7, MDA-MB-231, and MCF10A cells were incubated in growth media with the indicated concentration of $\mathrm{CoCl}_{2}$ for $24 \mathrm{~h}$. (A) Their phenotypes were photographed under a microscope. (B) The percentage of viable cells was evaluated by means of MTT assay, and the data are presented as the means \pm SD from three independent experiments $(\mathrm{P}<0.05)$. (C) Western blotting was used to measure TRIP-Br1 and TRIP-Br3 expression levels in different concentrations of $\mathrm{CoCl}_{2}$. Gene expression was also assessed with respect to apoptosis and autophagy (i.e., PARP, Bax, and XIAP for apoptosis and SQSTM1/p62 and LC3 for autophagy), with $\gamma$-tubulin serving as the loading control. Representative results from three independent experiments are shown.

representative cell lines for further studies. These were incubated in growth media with different concentrations of $\mathrm{CoCl}_{2}$ for $24 \mathrm{~h}$. The phenotypes of cell death in response to hypoxia were photographed under a microscope and the survival rates were measured (Fig. 2A and B). Our data showed that the rate of cell death was increased in response to $\mathrm{CoCl}_{2}$-induced hypoxia in a dose-dependent manner (Fig. 2B). Of note, the MCF7 and MDA-MB-231 cancer cells with high levels of
TRIP-Br1 protein survived for relatively longer periods of time in response to $\mathrm{CoCl}_{2}$ treatment, as compared with MCF10A normal cells (Fig. 2A and B).

In addition, our data also showed that hypoxia induced apoptosis and autophagy. These results were tested by means of western blot analysis with apoptosis-related regulatory proteins in the $\mathrm{CoCl}_{2}$-mediated oxygen-insufficient condition. In Fig. 2C, cleaved PARP and Bax expression levels 
D
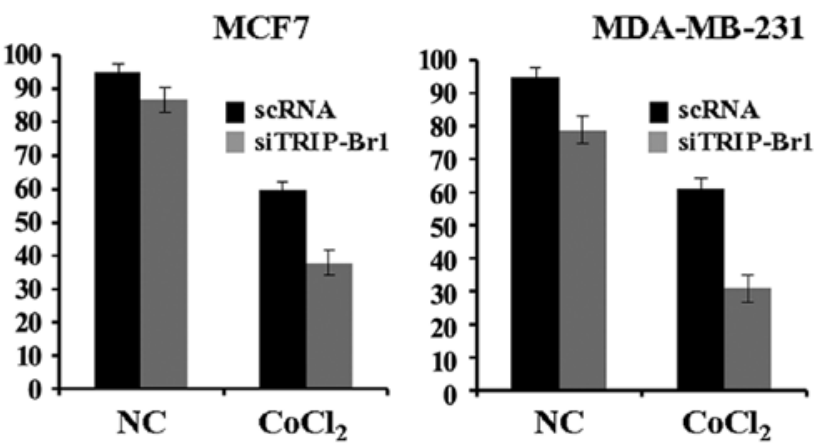

$\mathbf{E}$
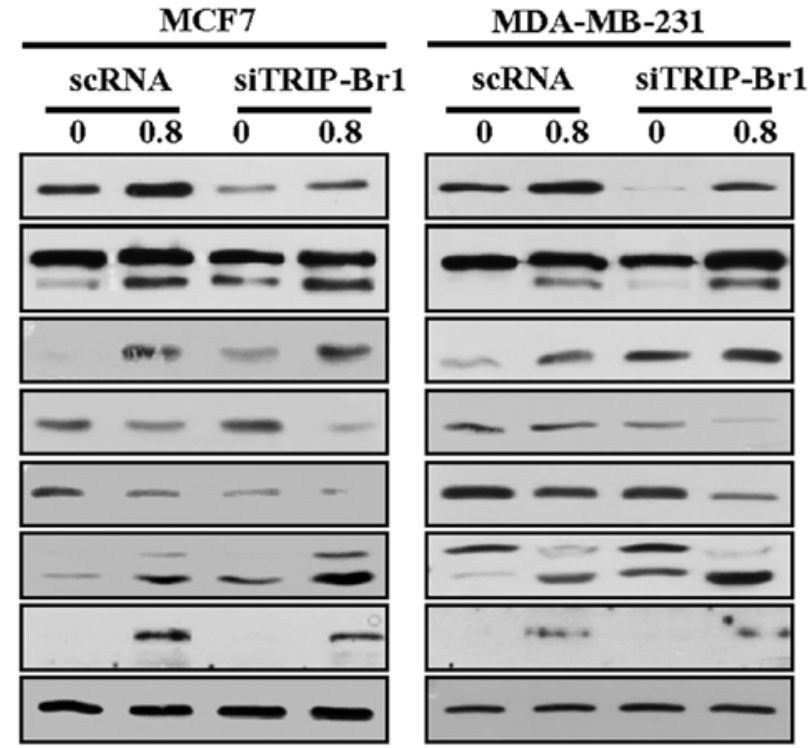

$\mathrm{CoCl}_{2}(\mathrm{mM})$
TRIP-Br1
PARP
Cleaved PARP
Bax
XIAP
SQSTM1/p62
LC3-I
LC3-II
HIF-1 $\alpha$
$\gamma$-tubulin

Figure 2 Continued. Inhibitory role of TRIP-Br1 on hypoxia-induced apoptosis. (D) MCF7 and MDA-MB-231 cancer cells were transiently transfected with scrambled RNA (scRNA) or TRIP-Brl silencing siRNA (siTRIP-Brl) and then incubated in growth media for $24 \mathrm{~h}$, the cells were then grown in the same media with or without $0.8 \mathrm{mM}$ of $\mathrm{CoCl}_{2}$ for $20 \mathrm{~h}$. The percentage of live cells was evaluated by means of MTT assay. (E) Cells were collected and subjected to western blot analysis using representative apoptosis and autophagy markers.

were increased in the MCF7, MDA-MB-231, and MCF10A cells in proportion to the $\mathrm{CoCl}_{2}$ concentration. In addition, hypoxia-stimulated autophagy was also examined because this phenomenon is important for achieving cellular homeostasis in response to a variety of stimuli including hypoxia $(12,15,41,42)$. The level of SQSTM1/p62 expression was decreased in response to $\mathrm{CoCl}_{2}$-induced hypoxia in a dose-dependent manner (Fig. 2C). The conversion ratio from LC3-I to LC3-II was enhanced in response to $\mathrm{CoCl}_{2}$-mediated hypoxia (Fig. 2C). These data obviously suggest that hypoxia can stimulate apoptosis and autophagy, in which TRIP-Br1 expression increased, implying an inhibitory role of TRIP-Br1 in apoptosis and autophagy.

Despite hypoxia being more toxic to cancer cells than to normal cells, many cancer cells are still able to surmount this stressful condition by modulating a cascade of regulatory systems or proteins. We showed that TRIP-Brl gene expression was significantly increased only in the cancer cells but not in the normal cells under $\mathrm{CoCl}_{2}$-evoked hypoxic condition. Our previous report also showed that TRIP-Brl provides an anti-apoptotic function to cancer cells in response to anticancer drugs and nutrient starvation $(25,34)$. Considering these results, it was hypothesized that TRIP-Br1 upregulation might contribute to the enhanced survival of cancer cells under hypoxic condition. This hypothesis is supported by the finding that TRIP-Brl knock-down in MCF7 and MDA-MB231 cancer cells accelerated cell death after they were exposed to $\mathrm{CoCl}_{2}$ (Fig. 2D). This possibility was also examined using western blot analysis, in which the levels of cleaved PARP and Bax expression increased after TRIP-Brl silencing in $\mathrm{CoCl}_{2}$ containing media and even in complete media (Fig. 2E). In our previous study, we proposed that TRIP-Brl could inhibit apoptosis by stabilizing the well-known inhibitor of apoptosis, X-linked inhibitor of apoptosis protein (XIAP) (25). We also showed that TRIP-Brl can suppress three types of programed cell deaths (apoptosis, autophagy, and necrosis/necroptosis) via XIAP stabilization (34). Therefore, XIAP expression levels were measured and found to be greatly decreased after TRIPBrl silencing in $\mathrm{CoCl}_{2}$-containing media, indicating that TRIP-Br1 can inhibit apoptosis by stabilizing XIAP (Fig. 2E). TRIP-Br1 knock-down also decreased SQSTM1/p62 protein levels and increased the LC3 conversion rate from LC3-I to LC3-II, indicating that TRIP-Br1 represses autophagy as well apoptosis under hypoxic condition (Fig. 2E).

Taken together, our data suggest that TRIP-Brl may function as an oncogene by rendering cancer cells resistant to apoptosis through the stabilization of XIAP under stressful hypoxic conditions. 


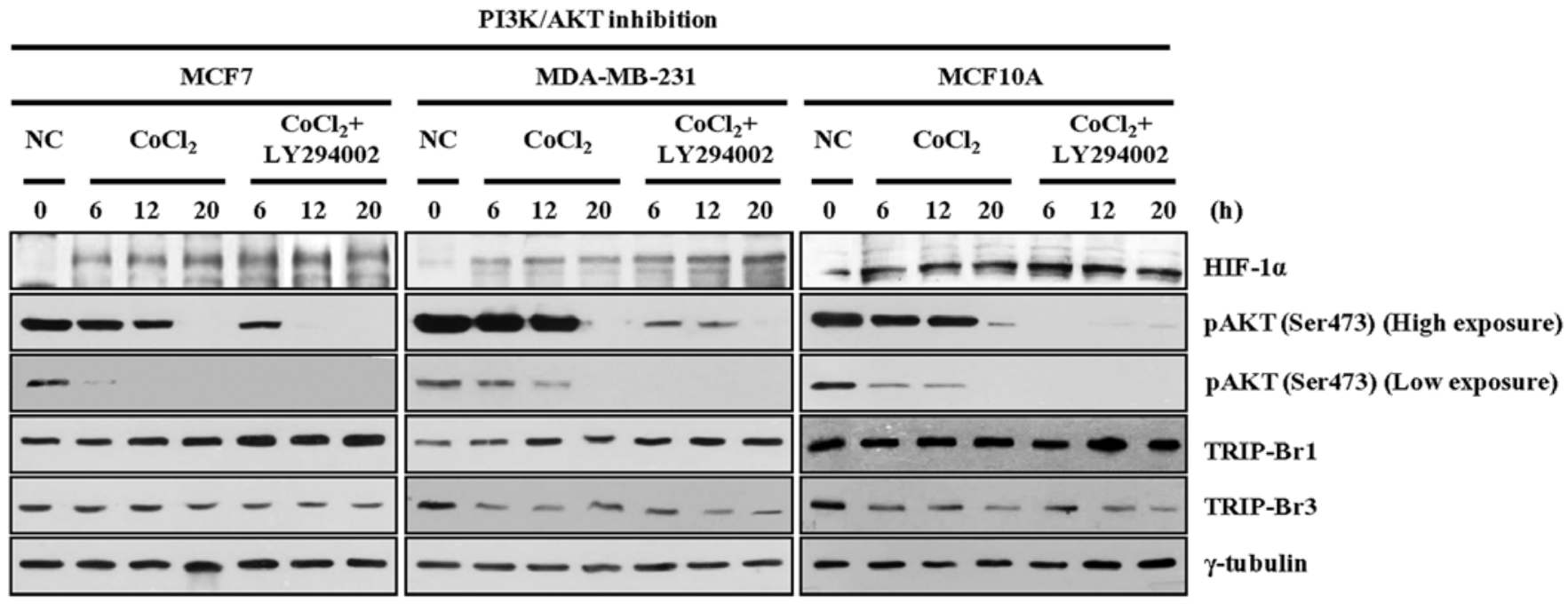

Figure 3. Effect of PI3K/AKT signaling pathway on TRIP-Br1 expression under $\mathrm{CoCl}_{2}$-induced hypoxic condition. MCF7, MDA-MB-231, and MCF10A cells were cultured in normal control growth media (NC), growth medium with $0.8 \mathrm{mM}$ of $\mathrm{CoCl}_{2}$, or growth medium with $0.8 \mathrm{mM}$ of $\mathrm{CoCl}_{2}$ and $20 \mu \mathrm{M}$ of LY294002 for the times indicated. Changes in TRIP-Br1 and TRIP-Br3 expression levels were measured by means of western blot analysis, as described in Materials and methods.

A

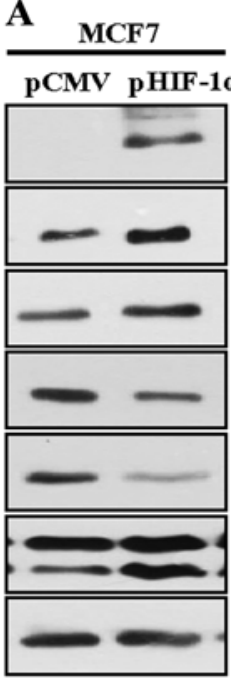

MDA-MB-231
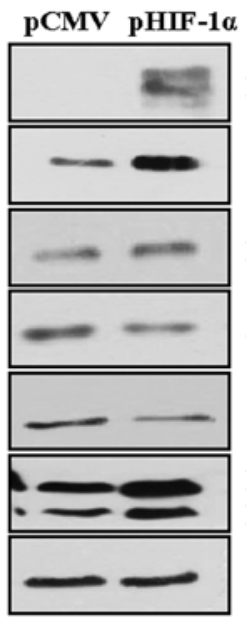

HIF-1a

TRIP-Br1

Bax

XIAP

SQSTM1/p62

LC3-I

LC3-II

$\gamma$-tubulin

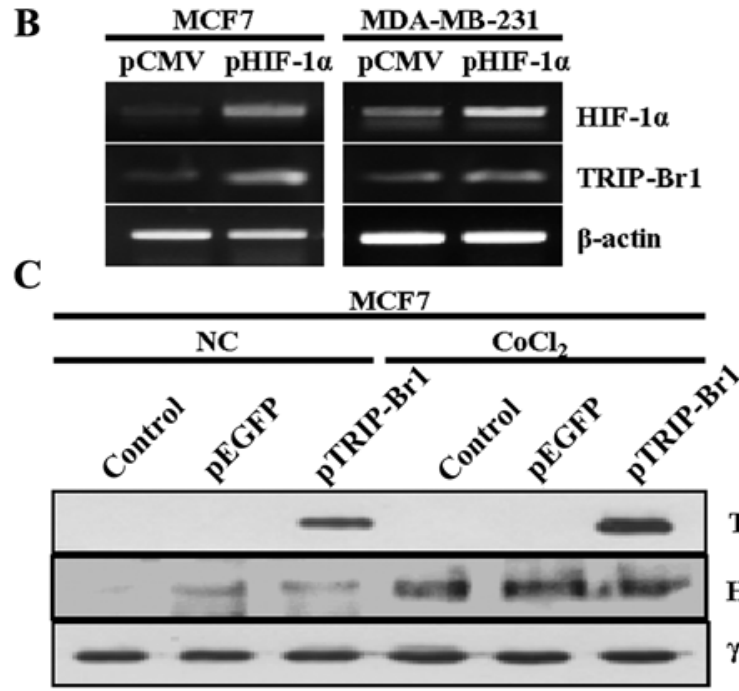

TRIP-Br1

HIF-1 $\alpha$

$\gamma$-tubulin

Figure 4. Effect of HIF-1 $\alpha$ on TRIP-Br1 expression. MCF7 and MDA-MB-231 cancer cells were transfected with a control vector (pCMV-tag2B) or HIF-10 overexpressing plasmid (pHIF-1 $\alpha$ ) (see Materials and methods) and the cells were incubated for $48 \mathrm{~h}$. Expression in each case was measured at the protein level (A) and the transcriptional level (B). $\gamma$-tubulin and $\beta$-actin were used as the control markers. (C) MCF7 cells were transfected with EGFP and EGFPTRIP-Br1 plasmids and were cultured for $24 \mathrm{~h}$ under conditions of normoxia and hypoxia $\left(0.8 \mathrm{mM} \mathrm{of} \mathrm{CoCl}_{2}\right)$. The level of HIF-1 $\alpha$ expression was measured by employing western blot analysis.

Effect of PI3K/AKT signaling pathway on TRIP-Brl expression under $\mathrm{CoCl}_{2}$-induced hypoxic condition. We have shown that hypoxia stimulated much higher levels of TRIP-Br1 expression in cancer cells as compared with normal cells, conferring on cancer cells the ability to develop an enhanced adaptive mechanism for resisting cell death. Our next question was what kind of mechanism is in charge of TRIP-Brl upregulation in response to hypoxia. It has been widely accepted that oncogenic protein usually stimulates cancer cell proliferation by triggering the activation of a string of signaling pathways, such as the phosphoinositol-3-kinase (PI3K)/AKT signaling pathway. We previously proposed that inhibition of the PI3K/ AKT signaling pathway increased TRIP-Brl but decreased
TRIP-Br3 expression under serum starvation condition $(34,37)$. Therefore, we examined the effect of this pathway on TRIP$\mathrm{Br} 1$ and TRIP-Br3 expression levels by treating MCF7, MDA-MB-231, and MCF10A cells with LY294002, a PI3K/ AKT inhibitor, in the media along with $\mathrm{CoCl}_{2}$. It was found that $\mathrm{CoCl}_{2}$-mediated hypoxia inhibited AKT phosphorylation on the 473 serine residue. Of note, inhibition of the PI3K/AKT pathway enhanced TRIP-Br1 upregulation or TRIP-Br3 downregulation under hypoxic condition (Fig. 3).

These results suggest that hypoxia-induced blockage of the PI3K/AKT signaling pathway is at least partly responsible for the changes in TRIP-Br1 and TRIP-Br3 expression levels in an oxygen-deprived environment. 
HIF-1 $\alpha$-mediated TRIP-Brl upregulation under hypoxic condition. It is well known that HIF- $1 \alpha$ is a central regulator of the expression of a broad range of genes, including those involved in tumorigenesis $(7,8,43,44)$. Thus, when we investigated whether or not HIF-1 $\alpha$ could affect oncogenic TRIP-Brl expression, we found that the transfection of HIF-1 $\alpha$ overexpressing plasmid into the MCF7 and MDA-MB-231 cells induced TRIP-Br1 upregulation, as was the case under hypoxic condition at both the protein and the transcriptional levels (Fig. 4A and B). Our data also revealed that HIF-1 $\alpha$ overexpression induced apoptosis and autophagy in the MCF7 and MDA-MB-231 cancer cells (Fig. 4A). In contrast, ectopic overexpression of the TRIP-Br1 gene had no effect on HIF-1 $\alpha$ expression (Fig. 4C).

These data suggest that HIF-1 $\alpha$ is at least partly responsible for the TRIP-Brl upregulation under hypoxic condition.

\section{Discussion}

Hypoxia has been a major topic in the area of cancer research for many decades. It is commonly found in many cases of solid tumors, in which the tumor cells struggle to adapt to this stressful environment by developing a broad range of cellular mechanisms $(7,8,12,15,18,44)$. In order to identify the mechanism to explain how cancer cells can survive and continue to grow despite being deprived of oxygen, we initially focused on the role of TRIP-Br1 oncoprotein in hypoxia.

Under oxygen-poor conditions, TRIP-Br1 was found to be significantly upregulated in the breast cancer cell lines selected for testing, but was only slightly upregulated in the normal cell lines. This finding underlies the fact that breast cancer cells and normal cells seem to react differently to hypoxia through the regulation of TRIP-Brl expression. In our study, oxygen deprivation caused injury to cancer and normal cells, inducing apoptosis. However, TRIP-Brl enables cancer cells to adapt to the stress of hypoxia, thus rending them resistant to cell death. Interestingly, even though TRIP-Br1 and TRIP-Br3 belong to the same TRIP-Br family, their levels of expression changed in opposite change in expression level under hypoxic conditions. These results imply the similar but different cellular functions of TRIP-Br1 and TRIP-Br3. TRIP-Br3 is known to function as a putative tumor suppressor, exerting cellular effects in tumorigenesis that differ from the effects of TRIP-Br1 (37-39). However, our previous study showed that both TRIP-Br1 and TRIP-Br3 can inhibit apoptosis in condition of serum starvation $(34,37)$. As we have now seen in the case of hypoxia, TRIP-Br1 expression levels increased while TRIP-Br3 expression decreased (37). In normal cells, TRIP-Br3 proteins are rapidly and greatly degraded. Rapid decrease in TRIP-Br3 expression triggers the ubiquitination and degradation of XIAP protein, eventually leading to cell death (37). In cancer cells, however, TRIP-Br3 expression is slightly downregulated (37). Therefore, we proposed that TRIP-Br3 and TRIP-Br1 may coordinately regulate apoptosis in normal and cancer cells by competitively interacting with XIAP. Considering our data as a whole, we speculate that TRIP-Br1 and TRIP-Br3 may be under a similar regulatory control system in hypoxic stressful environments. We also suspect that they may act as adopter proteins, functioning differently by changing their binding partners, although this possibility needs to be studied further.
Attention has also been focused on the effect of HIF-1 $\alpha$ on TRIP-Br1 expression, because the expression of many genes is known to be regulated by HIF-1 $\alpha$, a key regulator of a wide range of cellular responses to hypoxia in mammalian cells $(5,7,11,43)$. Our data showed that TRIP-Br1 expression was stimulated by HIF-1 $\alpha$ induction in both $\mathrm{CoCl}_{2}$-caused hypoxia and exogenous insertion at the transcriptional and translational levels. This finding provides a clue to the notion that HIF-1 $\alpha$ may contribute to the TRIP-Br1-mediated resistance of tumor cells to apoptosis caused by hypoxia. Nevertheless, it remains to be determined how HIF-1 $\alpha$ promotes TRIP-Br1 upregulation, directly or indirectly, under conditions of oxygen insufficiency.

In summary, our results demonstrate that TRIP-Br1 confers resistance to hypoxia-induced cell death in cancer cells. Thus, targeting TRIP-Brl-mediated cell death under hypoxic condition may provide vital information to those working in cancer research and in the development of effective anticancer drugs.

\section{Acknowledgements}

This work was supported by the grant from Sookmyung Women's University (2013).

\section{References}

1. Leontieva OV and Blagosklonny MV: Yeast-like chronological senescence in mammalian cells: Phenomenon, mechanism and pharmacological suppression. Aging (Albany, NY) 3: 1078-1091, 2011.

2. Rubin H: Multistage carcinogenesis in cell culture. Dev Biol (Basel) 106: 61-66; discussion 67, 143-160, 2001.

3. Hanahan D and Weinberg RA: Hallmarks of cancer: The next generation. Cell 144: 646-674, 2011.

4. Piret JP, Mottet D, Raes $\mathrm{M}$ and Michiels $\mathrm{C}$ : $\mathrm{CoCl}_{2}$, a chemical inducer of hypoxia-inducible factor-1, and hypoxia reduce apoptotic cell death in hepatoma cell line HepG2. Ann NY Acad Sci 973: 443-447, 2002.

5. Semenza GL: HIF-1: mediator of physiological and pathophysiological responses to hypoxia. J Appl Physiol (1985) 88: 1474-1480, 2000

6. Lee KA, Roth RA and LaPres JJ: Hypoxia, drug therapy and toxicity. Pharmacol Ther 113: 229-246, 2007.

7. Carmeliet P, Dor Y, Herbert JM, Fukumura D, Brusselmans K, Dewerchin M, Neeman M, Bono F, Abramovitch R, Maxwell P, et al: Role of HIF-1alpha in hypoxia-mediated apoptosis, cell proliferation and tumour angiogenesis. Nature 394: 485-490, 1998.

8. Rankin EB and Giaccia AJ: The role of hypoxia-inducible factors in tumorigenesis. Cell Death Differ 15: 678-685, 2008.

9. Rofstad EK and Danielsen T: Hypoxia-induced metastasis of human melanoma cells: Involvement of vascular endothelial growth factor-mediated angiogenesis. Br J Cancer 80: 1697-1707, 1999.

10. Kothari S, Cizeau J, McMillan-Ward E, Israels SJ, Bailes M Ens K, Kirshenbaum LA and Gibson SB: BNIP3 plays a role in hypoxic cell death in human epithelial cells that is inhibited by growth factors EGF and IGF. Oncogene 22: 4734-4744, 2003.

11. Suzuki H, Tomida A and Tsuruo T: Dephosphorylated hypoxiainducible factor 1alpha as a mediator of p53-dependent apoptosis during hypoxia. Oncogene 20: 5779-5788, 2001.

12. Tafani M, Schito L, Anwar T, Indelicato M, Sale P, Di Vito M, Morgante E, Beraldi R, Makovec F, Letari O, et al: Induction of autophagic cell death by a novel molecule is increased by hypoxia. Autophagy 4: 1042-1053, 2008.

13. Scarlatti F, Granata R, Meijer AJ and Codogno P: Does autophagy have a license to kill mammalian cells? Cell Death Differ 16: 12-20, 2009.

14. Bursch W, Karwan A, Mayer M, Dornetshuber J, Fröhwein U, Schulte-Hermann R, Fazi B, Di Sano F, Piredda L, Piacentini M, et al: Cell death and autophagy: Cytokines, drugs, and nutritional factors. Toxicology 254: 147-157, 2008. 
15. Zhang H, Bosch-Marce M, Shimoda LA, Tan YS, Baek JH, Wesley JB, Gonzalez FJ and Semenza GL: Mitochondrial autophagy is an HIF-1-dependent adaptive metabolic response to hypoxia. J Biol Chem 283: 10892-10903, 2008.

16. Ou XM, Chen K and Shih JC: Monoamine oxidase A and repressor R1 are involved in apoptotic signaling pathway. Proc Natl Acad Sci USA 103: 10923-10928, 2006.

17. Izuishi K, Kato K, Ogura T, Kinoshita T and Esumi $H$ : Remarkable tolerance of tumor cells to nutrient deprivation: Possible new biochemical target for cancer therapy. Cancer Res 60: 6201-6207, 2000.

18. Piret JP, Lecocq C, Toffoli S, Ninane N, Raes M and Michiels C: Hypoxia and $\mathrm{CoCl}_{2}$ protect HepG2 cells against serum deprivation- and t-BHP-induced apoptosis: A possible anti-apoptotic role for HIF-1. Exp Cell Res 295: 340-349, 2004.

19. Heyman SN, Leibowitz D, Mor-Yosef Levi I, Liberman A, Eisenkraft A, Elcalai R, Khamaisi $\mathrm{M}$ and Rosenberger $\mathrm{C}$ : Adaptive response to hypoxia and remote ischemia preconditioning: A new HIF era in clinical medicine. Acta Physiol (Oxf): Oct 9, 2015 (Epub ahead of print).

20. Hirst DG and Wood PJ: The adaptive response of mouse tumours to anaemia and retransfusion. Int J Radiat Biol Relat Stud Phys Chem Med 51: 597-609, 1987.

21. Acker T and Plate KH: A role for hypoxia and hypoxia-inducible transcription factors in tumor physiology. J Mol Med Berl 80 562-575, 2002.

22. Hsu SI, Yang CM, Sim KG, Hentschel DM, O'Leary E and Bonventre JV: TRIP-Br: A novel family of PHD zinc finger- and bromodomain-interacting proteins that regulate the transcriptional activity of E2F-1/DP-1. EMBO J 20: 2273-2285, 2001

23. Sim KG, Zang Z, Yang CM, Bonventre JV and Hsu SI: TRIP-Br links E2F to novel functions in the regulation of cyclin $E$ expression during cell cycle progression and in the maintenance of genomic stability. Cell Cycle 3: 1296-1304, 2004.

24. Sim KG, Cheong JK and Hsu SI: The TRIP-Br family of transcriptional regulators is essential for the execution of cyclin E-mediated cell cycle progression. Cell Cycle 5: 1111-1115, 2006.

25. Hong SW, Kim CJ, Park WS, Shin JS, Lee SD, Ko SG, Jung SI, Park IC, An SK, Lee WK, et al: p34SEI-1 inhibits apoptosis through the stabilization of the X-linked inhibitor of apoptosis protein: p34SEI-1 as a novel target for anti-breast cancer strategies. Cancer Res 69: 741-746, 2009.

26. Li J, Muscarella P, Joo SH, Knobloch TJ, Melvin WS, Weghorst CM and Tsai MD: Dissection of CDK4-binding and transactivation activities of p34(SEI-1) and comparison between functions of p34(SEI-1) and p16(INK4A). Biochemistry 44: 13246-13256, 2005

27. Sugimoto M, Nakamura T, Ohtani N, Hampson L, Hampson IN, Shimamoto A, Furuichi Y, Okumura K, Niwa S, Taya Y, et al Regulation of CDK4 activity by a novel CDK4-binding protein, p34(SEI-1). Genes Dev 13: 3027-3033, 1999.

28. Liew CW, Boucher J, Cheong JK, Vernochet C, Koh HJ, Mallol C, Townsend K, Langin D, Kawamori D, Hu J, et al: Ablation of TRIP-Br2, a regulator of fat lipolysis, thermogenesis and oxidative metabolism, prevents diet-induced obesity and insulin resistance. Nat Med 19: 217-226, 2013.
29. Fernandez-Marcos PJ, Pantoja C, Gonzalez-Rodriguez A, Martin N, Flores JM, Valverde AM, Hara E and Serrano M: Normal proliferation and tumorigenesis but impaired pancreatic function in mice lacking the cell cycle regulator seil. PLoS One 5: e8744, 2010

30. Jung S, Ohk J, Jeong D, Li C, Lee S, Duan J, Kim C, Lim JS, Yang Y, Kim KI, et al: Distinct regulatory effect of the p34SEI-1 oncoprotein on cancer metastasis in HER2/neu-positive and -negative cells. Int J Oncol 45: 189-196, 2014.

31. Hong SW, Shin JS, Lee YM, Kim DG, Lee SY, Yoon DH, Jung SY, Hwang JJ, Lee SJ, Cho DH, et al: p34 (SEI-1) inhibits ROS-induced cell death through suppression of ASK1. Cancer Biol Ther 12: 421-426, 2011.

32. Gozuacik D and Kimchi A: Autophagy as a cell death and tumor suppressor mechanism. Oncogene 23: 2891-2906, 2004.

33. Chen S, Rehman SK, Zhang W, Wen A, Yao L and Zhang J: Autophagy is a therapeutic target in anticancer drug resistance. Biochim Biophys Acta 1806: 220-229, 2010.

34. Jung S, Li C, Duan J, Lee S, Kim K, Park Y, Yang Y, Kim KI, Lim JS, Cheon CI, et al: TRIP-Br1 oncoprotein inhibits autophagy, apoptosis, and necroptosis under nutrient/serum-deprived condition. Oncotarget 6: 29060-29075, 2015.

35. Jung S, Li C, Jeong D, Lee S, Ohk J, Park M, Han S, Duan J, Kim C, Yang Y, et al: Oncogenic function of p34SEI-1 via NEDD4-1-mediated PTEN ubiquitination/degradation and activation of the PI3K/AKT pathway. Int J Oncol 43: 1587-1595, 2013.

36. Choi EJ, Kim T and Lee MS: Pro-apoptotic effect and cytotoxicity of genistein and genistin in human ovarian cancer SK-OV-3 cells. Life Sci 80: 1403-1408, 2007.

37. Li C, Jung S, Lee S, Jeong D, Yang Y, Kim KI, Lim JS, Cheon CI, Kim C, Kang YS, et al: Nutrient/serum starvation derived TRIP-Br3 down-regulation accelerates apoptosis by destabilizing XIAP. Oncotarget 6: 7522-7535, 2015.

38. Hayashi R, Goto Y, Ikeda R, Yokoyama KK and Yoshida K: CDCA4 is an E2F transcription factor family-induced nuclear factor that regulates E2F-dependent transcriptional activation and cell proliferation. J Biol Chem 281: 35633-35648, 2006.

39. Tategu M,Nakagawa H,Hayashi R and Yoshida K: Transcriptional co-factor CDCA4 participates in the regulation of JUN oncogene expression. Biochimie 90: 1515-1522, 2008.

40. Bennetts JS, Fowles LF, Berkman JL, van Bueren KL, Richman JM, Simpson F and Wicking C: Evolutionary conservation and murine embryonic expression of the gene encoding the SERTA domain-containing protein CDCA4 (HEPP). Gene 374: 153-165, 2006.

41. Bohensky J, Shapiro IM, Leshinsky S, Terkhorn SP, Adams CS and Srinivas V: HIF-1 regulation of chondrocyte apoptosis: Induction of the autophagic pathway. Autophagy 3: 207-214, 2007.

42. Pursiheimo JP, Rantanen K, Heikkinen PT, Johansen T and Jaakkola PM: Hypoxia-activated autophagy accelerates degradation of SQSTM1/p62. Oncogene 28: 334-344, 2009.

43. Weidemann A and Johnson RS: Biology of HIF-1alpha. Cell Death Differ 15: 621-627, 2008.

44. Greijer AE and van der Wall E: The role of hypoxia inducible factor 1 (HIF-1) in hypoxia induced apoptosis. J Clin Pathol 57: 1009-1014, 2004 